\title{
The Exterior Algebra and Central Notions in Mathematics
}

\section{Gunnar Fløystad}

\author{
Dedicated to Stein Arild Strømme (1951-2014)
}

The neglect of the exterior algebra is the mathematical tragedy of our century.

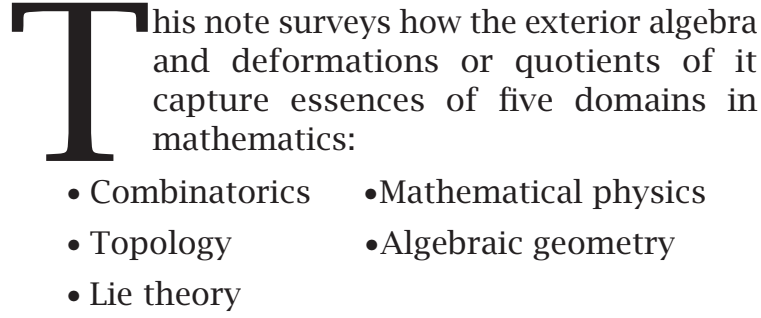

The exterior algebra originated in the work of Hermann Grassmann (1809-1877) in his book Ausdehnungslehre from 1844, and the thoroughly revised 1862 version, which now exists in an English translation [20] from 2000. Grassmann worked as a professor at the gymnasium in Stettin, then Germany. Partly because Grassmann was an original thinker and maybe partly because his education had not focused much on mathematics, the first edition of his book had a more philosophical than mathematical form and therefore gained little influence in the mathematical community. The second (1862) version was strictly mathematical. Nevertheless, it also gained little influence, perhaps because it had swung too far to the other side and was scarce of motivation. Over four hundred pages it developed the exterior and interior product and

Gunnar Fløystad is professor of mathematics at the University of Bergen, Norway. His email address is gunnar@mi . uib. no.

DOI: http://dx.doi.org/10.1090/noti1234 the somewhat lesser-known regressive product on the exterior algebra, which intuitively corresponds to intersection of linear spaces. It relates this to geometry and it also shows how analysis may be extended to functions of extensive quantities. Only in the last two decades of the 1800s did publications inspired by Grassmann's work achieve a certain mass. It may have been with some regret that Grassmann in his second version had an exclusively mathematical form, since he in the foreword says "[extension theory] is not simply one among the other branches of mathematics, such as algebra, combination theory or function theory, bur rather surpasses them, in that all fundamental elements are unified under this branch, which thus as it were forms the keystone of the entire structure of mathematics."

The present note indicates that he was not quite off the mark here. We do not make any further connections to Grassmann's original presentation, but rather present the exterior algebra in an entirely modern setting. For more on the historical context of Grassmann, see the excellent history of vector analysis [7], as well as proceedings from conferences on Grassmann's many-faceted legacy [41] and [38]. The last fifteen years have also seen a flurry of books advocating the very effective use of the exterior algebra and its derivation, the Clifford algebra, in physics, engineering, and computer 
science. In the last section we report briefly on this.

\section{The Exterior Algebra Concrete Definition}

Given a set $\left\{e_{1}, \ldots, e_{n}\right\}$ with $n$ elements, consider the $2^{n}$ expressions $e_{i_{1}} \wedge e_{i_{2}} \wedge \cdots \wedge e_{i_{r}}$ (here $\wedge$ is just a place separator), where the $i_{1}, i_{2}, \ldots, i_{r}$ are strictly increasing subsequences of $1,2, \ldots, n$. From this we form the vector space $E(n)$ over a field $\mathbb{k}$ with these expressions as basis elements.

Example 1. When $n=3$, the following eight expressions form a basis for $E(3)$ over the field $\mathbb{k}$ :

$1, e_{1}, e_{2}, e_{3}, e_{1} \wedge e_{2}, e_{1} \wedge e_{3}, e_{2} \wedge e_{3}, e_{1} \wedge e_{2} \wedge e_{3}$.

The element $e_{i_{1}} \wedge e_{i_{2}} \wedge \cdots \wedge e_{i_{r}}$ is considered to have degree $r$, so we get a graded vector space $E(n)$. Now we equip this vector space with a multiplication which we also denote by $\wedge$. The basic rules for this multiplication are
i) $e_{i} \wedge e_{i}=0$,
ii) $e_{i} \wedge e_{j}=-e_{j} \wedge e_{i}$.

These rules, together with the requirement that $\wedge$ be associative, i.e.,

$$
\text { iii) }(a \wedge b) \wedge c=a \wedge(b \wedge c)
$$

for all $a, b, c$ in $E(n)$, and linear, i.e.

$$
\text { iv) } a \wedge(\beta b+\gamma c)=\beta a \wedge b+\gamma a \wedge c
$$

for all $\beta, \gamma$ in the field $\mathbb{k}$ and $a, b, c$ in $E(n)$, determine the algebra structure on $E(n)$. For instance,

$$
\begin{aligned}
e_{5} \wedge\left(e_{1} \wedge e_{3}\right) & =e_{5} \wedge e_{1} \wedge e_{3} \\
& =-e_{1} \wedge e_{5} \wedge e_{3}\left(\text { switch } e_{5} \text { and } e_{1}\right) \\
& =e_{1} \wedge e_{3} \wedge e_{5} \quad\left(\text { switch } e_{5} \text { and } e_{3}\right) .
\end{aligned}
$$

\section{Abstract Definition}

Here we define the exterior algebra using standard machinery from algebra. Let $V$ be a vector space over $\mathbb{k}$, and denote by $V^{\otimes p}$ the $p$-fold tensor product $V \otimes_{\mathbb{k}} V \otimes_{\mathbb{k}} \cdots \otimes_{\mathbb{k}} V$. The free associative algebra on $V$ is the tensor algebra $T(V)=\bigoplus_{p \geq 0} V^{\otimes p}$ which comes with the natural concatenation product

$\left(v_{1} \otimes \cdots \otimes v_{r}\right) \cdot\left(w_{1} \otimes \cdots \otimes w_{S}\right)=v_{1} \otimes \cdots v_{r} \otimes w_{1} \otimes \cdots \otimes w_{S}$.

Let $R$ be the vector subspace of $V \otimes_{\mathbb{k}} V$ generated by all elements $v \otimes \nu$ where $v \in V$. The exterior algebra is the quotient algebra of $T(V)$ by the relations $R$. More formally, let $\langle R\rangle$ be the two-sided ideal in $T(V)$ generated by $R$. The exterior algebra $E(V)$ is the quotient algebra $T(V) /\langle R\rangle$. The product in this quotient algebra is commonly denoted by $\wedge$. Let $e_{1}, \ldots, e_{n}$ be a basis for $V$. We then have $e_{i} \wedge e_{i}=0$, since $e_{i} \otimes e_{i}$ is a relation in $R$. Similarly, $\left(e_{i}+e_{j}\right) \wedge\left(e_{i}+e_{j}\right)$ is zero. Expanding this

$$
0=e_{i} \wedge e_{i}+e_{i} \wedge e_{j}+e_{j} \wedge e_{i}+e_{j} \wedge e_{j},
$$

we see that $e_{i} \wedge e_{j}=-e_{j} \wedge e_{i}$. In fact, we obtain $v \wedge w+w \wedge v=0$ for any $v, w$ in $V$. Hence when the characteristic of $\mathbb{k}$ is not 2, the exterior algebra may be defined as $T(V) /\left\langle S_{2} V\right\rangle$ where

$$
S_{2} V=\{v \otimes w+w \otimes v \mid v, w \in V\}
$$

are the symmetric two-tensors in $V \otimes V$. The $p$ th graded piece of $E(V)$, which is the image of $V^{\otimes p}$, is denoted as $\wedge^{p} V$.

We shall in the following indicate:

- How central notions in various areas in mathematics arise from natural structures on the exterior algebra.

- How the exterior algebra or variations thereof are a natural tool in these areas.

\section{Combinatorics I: Simplicial Complexes and Face Rings}

For simplicity denote the set $\{1,2, \ldots, n\}$ as $[n]$. Each subset $\left\{i_{1}, \ldots, i_{r}\right\}$ of $[n]$ corresponds to a monomial $e_{i_{1}} \wedge e_{i_{2}} \wedge \cdots \wedge e_{i_{r}}$ in the exterior algebra $E(n)$. For instance, $\{2,5\} \subseteq[6]$ gives the monomial $e_{2} \wedge e_{5}$. It also gives the indicator vector $(0,1,0,0,1,0) \in \mathbb{Z}_{2}^{6}$ (where $\mathbb{Z}_{2}=\{0,1\}$ ), with 1's at positions 2 and 5 . We may then consider $e_{2} \wedge e_{5}$ to have this multidegree. This one-toone correspondence between subsets of $[n]$ and monomials in $E(n)$ suggests that it can be used to encode systems of subsets of a finite set. The set systems naturally captured by virtue of $E(n)$ being an algebra are the combinatorial simplicial complexes. These are families of subsets $\Delta$ of $[n]$ such that if $X$ is in $\Delta$, then any subset $Y$ of $X$ is also in $\Delta$.

Example 2. Let $n=6$. The sets

$$
\{1,2\},\{3,4\},\{3,5\},\{4,5,6\},
$$

together with all the subsets of each of these four sets, form a combinatorial simplicial complex.

The point of relating these to the algebra $E(n)$ is that combinatorial simplicial complexes on $[n]$ are in one-to-one correspondence with $\mathbb{Z}_{2}^{n}$-graded ideals $I$ in $E(n)$ or equivalently with $\mathbb{Z}_{2}^{n}$-graded quotient rings $E(n) / I$ of $E(n)$ : To a simplicial complex $\Delta$ corresponds the monomial ideal $I_{\Delta}$ generated by

$$
\left\{e_{i_{1}} \wedge \cdots \wedge e_{i_{r}} \mid\left\{i_{1}, \ldots, i_{r}\right\} \notin \Delta\right\} .
$$

Note that the monomials $e_{i_{1}} \wedge \cdots \wedge e_{i_{p}}$ with $\left\{i_{1}, \ldots, i_{p}\right\}$ in $\Delta$ then constitute a vector space basis for the quotient algebra $E(\Delta)=E(V) / I_{\Delta}$. We call this algebra the exterior face ring of $\Delta$.

For the simplicial complex in the example above, $E(\Delta)$ has a basis:

- degree 0: 1 ,

- degree 1: $e_{1}, e_{2}, e_{3}, e_{4}, e_{5}, e_{6}$, 
- degree 2: $e_{1} \wedge e_{2}, e_{3} \wedge e_{4}, e_{3} \wedge e_{5}, e_{4} \wedge e_{5}, e_{4} \wedge$ $e_{6}, e_{5} \wedge e_{6}$,

- degree 3: $e_{4} \wedge e_{5} \wedge e_{6}$.

Although subsets $\left\{i_{1}, \ldots, i_{r}\right\}$ of $[n]$ most naturally correspond to monomials in $E(n)$, one can also consider the monomial $x_{i_{1}} \cdots x_{i_{r}}$ in the polynomial ring $\mathbb{k}\left[x_{1}, \ldots, x_{n}\right]$. (Note, however, that monomials in this ring naturally correspond to multisets rather than to sets.) If one associates to $\Delta$ the analog monomial ideal in this polynomial ring, the quotient ring $\mathbb{k}[\Delta]$ is the Stanley-Reisner ring or simply the face ring of $\Delta$.

This opens up the arsenal of algebra to study $\Delta$. The study of $E(\Delta)$ and $\mathbb{k}[\Delta]$ has particularly centered around their minimal free resolutions and all the invariants that arise from such. The study of $\mathbb{k}[\Delta]$ was launched around 1975 with a seminal paper by Hochster [29] and Stanley's proof of the Upper Bound Conjecture for simplicial spheres; see [44]. Although one might say that $E(\Delta)$ is a more natural object associated to $\Delta, \mathbb{k}[\Delta]$ has been preferred for two reasons: (i) minimal free resolutions over $\mathbb{k}\left[x_{1}, \ldots, x_{n}\right]$ are finite in contrast to over the exterior algebra $E(n)$, (ii) $\mathbb{k}[\Delta]$ is commutative and the machinery for commutative rings is very well developed.

Since 1975 this has been a very active area of research, with various textbooks published: [44], [6], [34], and [22]. For the exterior face ring, see [16].

\section{Topology}

Let $u_{i}=(0, \ldots, 0,1,0, \ldots, 0)$ be the $i$ th unit coordinate vector in $\mathbb{R}^{n}$. To a subset $\left\{i_{1}, \ldots, i_{r}\right\}$ of $[n]$ we may associate the $(r-1)$-dimensional simplex which is the convex hull of the points $u_{i_{1}}, \ldots, u_{i_{r}}$ in $\mathbb{R}^{n}$. For instance, $\{2,3,5\} \subseteq[6]$ gives the simplex consisting of all points $\left(0, \lambda_{2}, \lambda_{3}, 0, \lambda_{5}, 0\right)$ in $\mathbb{R}^{6}$, where $\lambda_{i} \geq 0$ and $\lambda_{2}+\lambda_{3}+\lambda_{5}=1$.

A combinatorial simplicial complex $\Delta$ has a natural topological realization $X=|\Delta|$. It is the union of all the simplices in $\mathbb{R}^{n}$ associated to the sets $\left\{i_{1}, \ldots, i_{r}\right\}$ in $\Delta$.

Example 3. The simplicial complex given in Example 2 has a topological realization which may be pictured as:
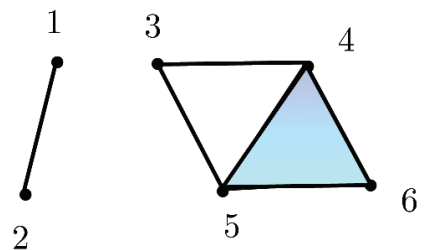

This is the disjoint union of a line segment and a disc with a handle.
We then say that $\Delta$ gives a triangulation of the space $X$. Now we can equip $E(n)$ with a differential $d$ of degree 1 by multiplying with $u=e_{1}+e_{2}+\cdots+e_{n}$. Then $d(a)=u \wedge a$, and this is a differential, since $d^{2}(a)=u \wedge u \wedge a=0$. The monomial $e_{i_{1}} \wedge e_{i_{2}} \wedge \cdots \wedge e_{i_{r}}$ of degree $r$ is then mapped to the degree $(r+1)$ sum:

$$
\sum_{i \notin\left\{i_{1}, \ldots, i_{r}\right\}} e_{i} \wedge e_{i_{1}} \wedge \cdots \wedge e_{i_{r}} .
$$

The face ring $E(\Delta)$ is a quotient of $E(n)$, and so we also obtain a differential on $E(\Delta)$. Letting $E(\Delta)^{r}$ be the degree $r$ part, this gives a complex

$$
E(\Delta)^{0} \stackrel{d^{0}}{\rightarrow} E(\Delta)^{1} \stackrel{d^{1}}{\rightarrow} E(\Delta)^{2} \stackrel{d^{2}}{\rightarrow} \cdots .
$$

From this complex and its dual we calculate the prime invariants in topology, the cohomology and homology of the topological space $X$. The cohomology is

$$
H^{i+1}(E(\Delta), d)=\tilde{H}^{i}(X, \mathbb{k}) \text { for } i \geq 0,
$$

where $\tilde{H}^{i}(X, \mathbb{k})$ is the reduced cohomology of $X$. (For $i>0$ this is simply the cohomology $H^{i}(X, \mathbb{k})$, while for $i=0$ this is the cokernel $H^{0}(p t, \mathbb{k}) \rightarrow H^{0}(X, \mathbb{k})$.) Dualizing the complex above we get $E(\Delta)^{*}$ as a subcomplex of $E(n)^{*}$ :

$$
\cdots \stackrel{\partial_{2}}{\rightarrow}\left(E(\Delta)^{*}\right)_{2} \stackrel{\partial_{1}}{\rightarrow}\left(E(\Delta)^{*}\right)_{1} \stackrel{\partial_{0}}{\rightarrow}\left(E(\Delta)^{*}\right)_{0} .
$$

Here $\left(E(\Delta)^{*}\right)_{r+1}$ has a basis consisting of monomials

$$
e_{i_{0}}^{*} \wedge \cdots \wedge e_{i_{r}}^{*}
$$

where $\left\{i_{0}, \ldots, i_{r}\right\}$ are the $r$-dimensional faces of the simplicial complex $\Delta$. The differential $\partial$ is contraction with the element $u$

$$
a \stackrel{\partial}{\mapsto} u \neg a,
$$

sending the monomial (1) to its boundary

$$
\sum_{j=0}^{d}(-1)^{j} e_{e_{i_{0}}}^{*} \wedge \cdots \wedge \widehat{e_{i_{j}}^{*}} \wedge \cdots e_{i_{r}}^{*} .
$$

(Here $\widehat{e_{i_{j}}^{*}}$ means omitting this term.) The homology $H_{i}\left(E(\Delta)^{*}, \partial\right)$ computes the reduced simplicial homology of the space $X$ (but with a shift by one in homological index $i$ ). In Example 3 we get $H_{1}\left(E(\Delta)^{*}, \partial\right)=\mathbb{k}$ one-dimensional, one less than the number of components of $\Delta$, and $H_{2}\left(E(\Delta)^{*}, \partial\right)=\mathbb{k}$ one-dimensional, since there is a noncontractible 1-cycle through the points 3,4 and 5 .

A good introduction to algebraic topology, starting from simplicial complexes, is [35]. 


\section{Lie Theory}

Differential graded algebras (DGA) occur naturally in many areas. They provide the "full story" in contrast to graded algebras, which often are the cohomology of a DGA, like the cochain complex of a topological space, in contrast to its cohomology ring.

A DGA is graded algebra $A=\bigoplus_{p \geq 0} A_{p}$ with a differential $d$, i.e., $d^{2}=0$, which is a derivation; i.e., for homogeneous elements $a, b$ in $A$ it satisfies

$$
d(a \cdot b)=d(a) \cdot b+(-1)^{\operatorname{deg}(a)} a \cdot d(b) .
$$

The differential $d$ either has degree 1 or -1 according to whether it raises degrees by one or decreases degrees by one.

What does it mean to give a $\mathbb{k}$-linear differential $d$ of degree 1 on $E(V)$ such that $(E(V), d)$ becomes a DGA? Between degrees 1 and 2 we have a map

$$
V \stackrel{d}{\rightarrow} \wedge^{2} V
$$

By the definition of derivation above (2), it is easy to see that any linear map between these vector spaces extends uniquely to a derivation $d$ on $E(V)$. Denote by $\mathfrak{g}$ the dual vector space $V^{*}=\operatorname{Hom}_{k}(V, k)$. Dualizing the above map we get a map

$$
\begin{array}{rll}
\bigwedge^{2} \mathfrak{g} & \stackrel{d^{*}}{\rightarrow} & \mathfrak{g} \\
x \wedge y & \mapsto & {[x, y] .}
\end{array}
$$

It turns out that $d$ gives a differential, i.e., $d^{2}=0$, if and only if the map $d^{*}$ satisfies the Jacobi identity

$$
[x,[y, z]]+[y,[z, x]]+[z,[x, y]]=0 \text {. }
$$

Thus giving $E(V)$ the structure of a DGA with differential of degree 1 is precisely equivalent to giving $\mathfrak{g}=V^{*}$ the structure of a Lie algebra.

The cohomology of the complex $(E(V), d)$ computes the Lie algebra cohomology of $\mathfrak{g}$. If $\mathfrak{g}$ is the Lie algebra of a connected compact Lie group $G$ (over $\mathbb{k}=\mathbb{R}$ ), it is a theorem of Cartan [1, Cor.12.4], that the cohomology ring $H^{*}(E(V), d)$ is isomorphic to the cohomology ring $H^{*}(G, \mathbb{R})$. A good and comprehensive introduction to Lie algebras is [31]. The book [18] is much used as a reference book for the representations of semisimple Lie groups and Lie algebras. But there are many books on this, and the above are mentioned mostly because I learned from these books.

Now denote the dual $V^{*}$ by $W$, and by $S(W)=$ $\operatorname{Sym}(W)$ the symmetric algebra, i.e., the polynomial ring whose variables are any basis of $W$. The pair $E(V)$ and $S(W)$ is the prime example of a Koszul dual pair of algebras; see [39], [3] for the general framework of Koszul duality. Furthermore, when we equip $E(V)$ with the differential $d$, the pair $(E(V), d)$ can be considered as the Koszul dual of the enveloping algebra $U(\mathfrak{g})$ of the Lie algebra $\mathfrak{g}$; see [40], [17] for the Koszul duality in the differential graded setting. Koszul duality gives functors between the module categories of these algebras, which on suitable quotients of these give an equivalence of categories.

\section{Combinatorics II: Hyperplane Arrangements and the Orlik-Solomon Algebra}

Simplicial complexes are basic combinatorial structures, and we have seen in the section Combinatorics I how they are captured by the exterior face ring $E(\Delta)$. One of the most successful unifying abstractions in combinatorics is that of a matroid (a term giving more associations might be independence structures), which is a special type of a simplicial complex. To a matroid there is associated a quotient algebra of the exterior algebra with remarkable connections to hyperplane arrangements.

A prime source of matroids is linear algebra. Let us consider the vector space $\mathbb{k}^{m}$ and let $x_{1}, \ldots, x_{m}$ be coordinate functions on this space. A linear form $v=\sum \lambda_{j} x_{j}$ gives a hyperplane in $\mathbb{k}^{m}$ : the set of all points $\left(a_{1}, \ldots, a_{m}\right) \in \mathbb{k}^{m}$ such that $\sum_{j} \lambda_{j} a_{j}=0$. A set of linear forms $v_{1}, \ldots, v_{n}$ determines hyperplanes $H_{1}, H_{2}, \ldots, H_{n}$. We call this a hyperplane arrangement. It turns out that a number of essential properties of the hyperplane arrangement are determined by the linear dependencies between the linear forms $v_{1}, \ldots, v_{n}$. We get a combinatorial simplicial complex $M$ on $[n]$ consisting of all subsets $\left\{i_{1}, \ldots, i_{r}\right\}$ of $[n]$ such that $v_{i_{1}}, \ldots, v_{i_{r}}$ are linearly independent vectors. But there is more structure on this $M$, making it a matroid. A simplicial complex $M$ on $[n]$ is a matroid if the following extra condition holds:

If $X$ and $Y$ are independent sets of $M$, with the cardinality of $Y$ larger than that of $X$, there is $y \in$ $Y \backslash X$ such that $X \cup\{y\}$ is independent.

The elements of $M$ are called the independent sets of the matroid, while subsets of $[n]$ not in $M$ are dependent. The diversity which the abstract notion of a matroid captures is illustrated by the following examples, where we give independent sets of matroids:

- Linear independent subsets of a set of vectors $\left\{v_{1}, v_{2}, \ldots, v_{n}\right\}$.

- Edge sets of graphs which do not contain a cycle.

- Partial transversals of a family of sets $A_{1}, A_{2}, \ldots, A_{N}$.

Example 4. Consider the hyperplane arrangement in $\mathbb{C}^{2}$ given by the two coordinate functions $v_{1}=x_{1}$ and $v_{2}=x_{2}$. The complement $\mathbb{C}^{2} \backslash H_{1} \cup H_{2}$ consists of the pairs $(a, b)$ with nonzero coordinates; i.e., the complement is $\left(\mathbb{C}^{*}\right)^{2}$ where $\mathbb{C}^{*}=\mathbb{C} \backslash\{0\}$. Since $\mathbb{C}^{*}$ is homotopy equivalent to the circle $S^{1}$, the 
complement $\mathbb{C}^{2} \backslash H_{1} \cup H_{2}$ will be homotopy equivalent to the torus $S^{1} \times S^{1}$. The cohomology ring of this torus is the exterior algebra $E(2)$.

This example generalizes to a description of the cohomology ring of the complement of any hyperplane arrangement in $\mathbb{C}^{m}$. The matroid $M$ of the hyperplane arrangement, being a simplicial complex, gives by the section Combinatorics I a monomial ideal $I_{M}$ in $E(n)$. The dual element $u=e_{1}^{*}+\cdots+e_{n}^{*}$ gives a contraction $a \stackrel{\partial}{\longrightarrow} u \neg a$ sending $e_{i_{1}} \wedge \cdots \wedge e_{i_{r}}$ to

$$
\sum_{j}(-1)^{j} e_{i_{1}} \wedge \cdots \hat{e_{i_{j}}} \cdots \wedge e_{i_{r}}
$$

(here $\hat{e_{j}}$ means omitting this term). Now $I_{M}+\partial\left(I_{M}\right)$ also becomes an ideal in $E(n)$. The quotient $A(M)=E(n) /\left(I_{M}+\partial\left(I_{M}\right)\right)$ is called the OrlikSolomon algebra associated to the hyperplane arrangement. In 1980 Peter Orlik and Louis Solomon proved the following amazing result [36].

Theorem 5. Let $T=\mathbb{C}^{m} \backslash \bigcup_{i=1}^{n} H_{i}$ be the complement of a complex hyperplane arrangement. Then the algebra $A(M)$ is the cohomology ring $H^{*}(T, \mathbb{C})$.

Example 6. Consider $u_{1}=x_{1}-x_{2}, u_{2}=x_{2}-x_{3}$, and $u_{3}=x_{3}-x_{1}$ on $\mathbb{C}^{3}$. There is one dependency here, between $u_{1}, u_{2}$, and $u_{3}$. Thus the Orlik-Solomon algebra is $E$ (3) divided by the ideal generated by the relation

$$
\partial\left(e_{1} \wedge e_{2} \wedge e_{3}\right)=e_{1} \wedge e_{2}-e_{1} \wedge e_{3}+e_{2} \wedge e_{3} .
$$

The quotient algebra has dimensions 1,3 , and 2 in degrees 0,1 , and 2 respectively. For the complement $T=\mathbb{C}^{3} \backslash H_{1} \cup H_{2} \cup H_{3}$ we therefore have

$$
H^{0}(T, \mathbb{C})=\mathbb{C}, H^{1}(T, \mathbb{C})=\mathbb{C}^{3}, H^{2}(T, \mathbb{C})=\mathbb{C}^{2} .
$$

For hyperplane arrangements, or more generally, for matroids, the Orlik-Solomon algebra has been much studied recently, [47], [15]. The algebraic properties of the Orlik-Solomon algebra give a number of natural invariants for hyperplane arrangements.

\section{Mathematical Physics}

The Clifford algebra may be viewed as a deformation of the exterior algebra. The exterior algebra $E(V)$ is defined as the quotient algebra $T(V) /\left\langle S_{2} V\right\rangle$. Fix a symmetric bilinear form $b: S_{2} V \rightarrow \mathbb{k}$. Let $R=\left\{r-b(r) \mid r \in S_{2} V\right\}$. The Clifford algebra is the quotient of the tensor algebra by the relations $R$ :

$$
C l_{b}=T(V) /\langle R\rangle .
$$

Like the exterior algebra $E(V)=E(n)$ it has a basis consisting of all products $e_{i_{1}} \cdots \cdots e_{i_{r}}$ for subsets $\left\{i_{1}<\cdots<i_{r}\right\}$ of $\{1, \ldots, n\}$ and so is also of dimension $2^{n}$ as a vector space over $\mathbb{k}$. Note that we get the exterior algebra when the bilinear form $b=0$.
Clifford algebras are mostly applied when the field $\mathbb{k}$ is the real numbers $\mathbb{R}$. Let $V=\langle i\rangle$ be a one-dimensional vector space generated by a vector $i$, and let the quadratic form be given by $i^{2} \stackrel{b}{\mapsto}-1$. Then the associated Clifford algebra is the complex numbers. When $V=\langle i, j\rangle$ is a two-dimensional space and

$$
i \otimes i \stackrel{b}{\mapsto}-1, \quad i \otimes j+j \otimes i \stackrel{b}{\mapsto} 0, \quad j \otimes j \stackrel{b}{\mapsto}-1,
$$

we obtain the quaternions. In general, for a real symmetric form $b$, we may find a basis for $V$ such that if $x_{1}, \ldots, x_{n}$ are the coordinate functions, the form is

$$
\sum_{i=1}^{p} x_{i}^{2}-\sum_{i=p+1}^{n=p+q} x_{i}^{2}
$$

Such a Clifford algebra is denoted $C l_{p, q}$.

So $C l_{0,1}$ is the complex numbers and $C l_{0,2}$ is the quaternions. Clifford algebras have interesting periodic behaviour: $\mathrm{Cl}_{p+1, q+1}$ is isomorphic to the $2 \times 2$-matrices $\mathrm{M}_{2}\left(C l_{p, q}\right)$, and each of $C l_{p+8, q}$ and $\mathrm{Cl}_{p, q+8}$ is isomorphic to the $16 \times 16$-matrices $M_{16}\left(C l_{p, q}\right)$. Thus Clifford algebras over the reals are essentially classified by $C l_{p, 0}$ and $C l_{0, q}$ for $p$, $q \leq 7$. For a nice introduction to Clifford algebras, see [19].

When $C l_{p, q}$ is a simple algebra and $C l_{p, q} \rightarrow$ $E n d(W)$ is an irreducible representation of $C l_{p, q}$ then $W$ is called a spinor space. These representations occur a lot in mathematical physics. For instance, $C l_{1,3}$ is isomorphic to $M_{4}(\mathbb{R})$, and this representation on $\mathbb{R}^{4}$ is the Minkowski space with one time dimension and three space dimensions. A pioneer in the application of Clifford algebras in mathematical physics is David Hestenes [23], [24], [25], where he envisions the complete use of it in classical mechanics. He calls this geometric algebra. The book [8] offers a leisurely introduction to the application of geometric algebra in physics. Basil Hiley is another advocate for the algebraic approach to quantum mechanics [28]:

...that quantum phenomena per se can be entirely described in terms of Clifford algebras taken over the reals without the need to appeal to specific representations in terms of wave functions in a Hilbert space. This removes the necessity of using Hilbert space and all the physical imagery that goes with the use of the wave function.

\section{Algebraic Geometry}

Finitely generated graded modules over exterior algebras seem far removed from geometry. However, we shall see that they encode perhaps the most significant invariants of algebraic geometry, the cohomological dimensions of twists of sheaves on projective spaces. 
Example 7. Let $n=2$ and $E=E(2)$. Consider the map of free $E$-modules:

$$
E \stackrel{d=\left[\begin{array}{c}
e_{2} \\
e_{1}
\end{array}\right]}{\longrightarrow} E^{2} .
$$

Writing $E^{2}=E u_{1} \oplus E u_{2}$ where $u_{1}$ and $u_{2}$ are generators of this module, the cokernel of this map is a module $M=E u_{1} \oplus E u_{2} /\left\langle e_{2} u_{1}+e_{1} u_{2}\right\rangle$. Such a map may, as we shortly explain, be completed to a complex of free $E$-modules (we let $d^{0}=d$ ):

$$
\begin{aligned}
& \cdots \rightarrow E^{2} \stackrel{d^{-2}=\left[\begin{array}{ll}
e_{1} & e_{2}
\end{array}\right]}{\longrightarrow} E \frac{d^{-1}=\left[\begin{array}{ll}
e_{1} \wedge e_{2}
\end{array}\right]}{\longrightarrow} E \stackrel{d^{0}=\left[\begin{array}{l}
e_{2} \\
e_{1}
\end{array}\right]}{\longrightarrow} \\
& E^{2} \stackrel{d^{1}=\left[\begin{array}{cc}
e_{2} & 0 \\
e_{1} & e_{2} \\
0 & e_{1}
\end{array}\right]}{\longrightarrow} E^{3} \rightarrow E^{4} \rightarrow \cdots
\end{aligned}
$$

It is a complex since $d^{p} \circ d^{p-1}=0$, as is easily verified. It is also exact at each place, meaning that the kernel of $d^{p}$ equals the image of $d^{p-1}$ for each $p$. Thus it is an acyclic complex.

Every finitely generated graded module $M$ over $E=E(V)$, or equivalently map $d$, gives rise to such an acyclic complex. In the example above the ranks of the free modules follow a simple pattern, $1,2,3,4, \ldots$, but in general, what are these ranks? Can they be given a meaningful interpretation? Indeed, a discovery from 2003, [13], tells us this is the case.

As a module over itself $E(V)$ is both a projective and an injective module. Given a finitely generated graded module $M$ over $E(V)$, one can make a minimal free (and so projective) resolution

$$
P^{\bullet} \rightarrow M, \quad \text { where } P^{p}=\bigoplus_{q \in \mathbb{Z}} W_{q}^{p} \otimes_{k} E
$$

(the $W_{q}^{p}$ are vector spaces over the field $\mathbb{k}$ whose elements are considered to have degree $q$ ) and a minimal injective resolution

$$
M \rightarrow I^{\bullet}, \quad \text { where } I^{p}=\bigoplus_{q \in \mathbb{Z}} W_{q}^{p} \otimes_{k} E
$$

and splice these together into an acyclic complex (as in Example 7),

(4) $\quad T: \cdots \rightarrow P^{-1} \rightarrow P^{0} \rightarrow I^{1} \rightarrow I^{2} \rightarrow \cdots$,

called the Tate resolution of $M$. So we get a correspondence:

(5) graded modules over $E(V)$

$$
\leadsto \text { Tate resolutions over } E(V) \text {. }
$$

Now let us pass to another construction starting from the finitely generated graded module $M=$ $\bigoplus_{i=a}^{b} M_{i}$ over the exterior algebra $E(V)$. Let $W$ be the dual vector space $V^{*}$. The multiplication $V \otimes_{k} M_{i} \rightarrow M_{i+1}$ gives a map

$$
M_{i} \rightarrow W \otimes_{\mathrm{k}} M_{i+1} \text {. }
$$

Let $S=\operatorname{Sym}(W)$ be the symmetric algebra (a polynomial ring). The map (6) gives rise to maps (7) $\cdots \rightarrow S \otimes_{\mathbb{k}} M_{p} \stackrel{d^{p}}{\longrightarrow} S \otimes_{\mathbb{k}} M_{p+1} \stackrel{d^{p+1}}{\longrightarrow} \cdots \stackrel{d^{q-1}}{\longrightarrow} S \otimes_{k} M_{q} \rightarrow \cdots$ These maps give a (bounded) complex of $S$-modules, i.e., $d^{i+1} \circ d^{i}=0$ (This is a correspondence within the framework of Koszul duality, mentioned in the section "Lie Theory".) Any finitely generated graded $S=\operatorname{Sym}(W)$-module may be sheafified to a coherent sheaf on the projective space $\mathbb{P}(W)$. In particular, we may sheafify the above complex and get a complex of coherent sheaves:

(8) graded modules over $E(V)$

$\leadsto$ bounded complexes of coherent sheaves on $\mathbb{P}(W)$.

This correspondence is from 1978 [4] and is the celebrated Bernstein-Gelfand-Gelfand (BGG) correspondence. Somewhat more refined, it may be described as an equivalence of categories between suitable categories of the objects in (8).

The amazing thing is that if $M$ via the BGGcorrespondence (8) gives a coherent sheaf $\mathcal{F}$ on $\mathbb{P}(W)$ (this means that the sheafification of the complex (7) has only one nonzero cohomology sheaf $\mathcal{F}$ ), then we can read off all the sheaf cohomology groups of all twists of $\mathcal{F}$ from the Tate resolution $T$ which we get via the correspondence (5).

Theorem 8 ([13, Thm. 4.1]). If $M$ via the $B G G$ correspondence (8) gives a coherent sheaf $\mathcal{F}$ on the projective space $\mathbb{P}(W)$, and the Tate resolution of $M$ is (4), then the sheaf cohomology

$$
H^{p}(\mathbb{P}(W), \mathcal{F}(q))=W_{q}^{p+q} .
$$

Returning to the initial Example 7 in this section, the sheaf corresponding to this module $M$ is the structure sheaf $\mathcal{O}_{\mathbb{P} 1}$ on the projective line $\mathbb{P}^{1}=\mathbb{P}(W)$. The Tate resolution (3) therefore tells us that for $d \geq 0$, the sheaf cohomology

$$
\begin{gathered}
H^{0}\left(\mathbb{P}^{1}, \mathcal{O}_{\mathbb{P}}(d)\right)= \begin{cases}\mathbb{k}^{d+1}, & d \geq 0, \\
0, & d<0,\end{cases} \\
H^{1}\left(\mathbb{P}^{1}, \mathcal{O}_{\mathbb{P}}(-d)\right)= \begin{cases}\mathbb{k}^{d-1}, & -d<0, \\
0, & -d \geq 0 .\end{cases}
\end{gathered}
$$

An important feature of a Tate resolution $T$ is that it is fully determined by an arbitrary differential $T^{i} \stackrel{d^{i}}{\longrightarrow} T^{i+1}$. This is because $T^{\leq i}$ is a minimal projective resolution of im $d^{i}$, and $T^{>i}$ is a minimal injective resolution of im $d^{i}$. This gives us incredible freedom in construction. An arbitrary homogeneous matrix $A$ of exterior forms gives a map

$$
\bigoplus_{q} W_{q}^{0} \otimes_{\mathrm{k}} E \stackrel{d_{A}}{\rightarrow} \bigoplus_{q} W_{q}^{1} \otimes_{\mathrm{k}} E
$$


The module $M=\operatorname{im} d_{A}$ over the exterior algebra then gives a complex of coherent sheaves $\mathcal{F}^{\bullet}$ on $\mathbb{P}(W)$ by (8), and all such bounded complexes on $\mathbb{P}(W)$ do (in a suitable sense) come from such a homogeneous matrix $A$ of exterior forms. Thus bounded complexes of coherent sheaves on projective spaces can be specified by giving a homogeneous matrix $A$ of exterior forms, and any matrix $A$ will give some such bounded complex. The Tate resolution associated to $M$ and $\mathcal{F}^{\bullet}$ is the complex we obtain by taking a minimal projective resolution of ker $d_{A}$ in (9) and a minimal injective resolution of coker $d_{A}$, and this resolution tells us the cohomology of the complex of coherent sheaves.

Example 9. Let $V$ be the five-dimensional vector space generated by $\left\{e_{1}, e_{2}, e_{3}, e_{4}, e_{5}\right\}$. The matrix

$$
A=\left[\begin{array}{lllll}
e_{1} \wedge e_{2} & e_{2} \wedge e_{3} & e_{3} \wedge e_{4} & e_{4} \wedge e_{5} & e_{5} \wedge e_{1} \\
e_{3} \wedge e_{5} & e_{4} \wedge e_{1} & e_{5} \wedge e_{2} & e_{1} \wedge e_{3} & e_{2} \wedge e_{4}
\end{array}\right]
$$

gives a map $E^{5} \rightarrow E^{2}$. Via the BGG-correspondence (8) this gives the celebrated Horrocks-Mumford bundle on $\mathbb{P}^{4}$ discovered over forty years ago [30], see also [13, Section 8]. In characteristic zero this is essentially the only known indecomposable rank two bundle on any projective space of dimension greater or equal to four. It is an intriguing problem to use the methods above to try to construct new bundles of rank $\leq n-2$ on a projective space $\mathbb{P}^{n}$, but to our knowledge nobody has yet been successful.

Tate resolutions and algebraic geometry are treated in the books [14] and [12]. The software program [21] contains the package BGG for doing computations with Tate resolutions.

\section{Modelling and Computations}

The last fifteen years have seen a flurry of books and treatises giving applications of exterior algebras and Clifford algebras, usually under the name "geometric algebra." Groups at the University of Cambridge and the University of Amsterdam have been particularly active in promoting geometric algebra. The book Geometric Algebra for Physicists [8] by C. Doran and A. Lasenby is a very well written and readable introduction to exterior algebras, Clifford algebras, and their applications in all areas of physics, following the ideas outlined by D. Hestenes. A more advanced treatment is [2]. The book Geometric Algebra for Computer Scientists: An Object Oriented Approach, [10] by L. Dorst, D. Fontijne, and S. Mann shows geometric algebra as an effective tool to describe a variety of geometric models involving linear spaces, circles, spheres, rotations, and reflections. In particular it considers the conformal geometric model developed in [32]. Geometric Algebra for Engineers, [37] by C.
Perwass similarly applies geometric algebra to models occurring in engineering: camera positions, motion tracking, and statistics. It also considers numerical aspects of its implementation. Other books on geometric algebra and its use in computer modelling and engineering are [26], [27], [43], [9], [45], and [46]. The book [11] gives a panorama of applications by a wide range of authors.

The comprehensive book Grassmann Algebra [5] considers all aspects of computations concerning the exterior algebra with Mathematica. It treats the exterior, interior, and regressive products and geometric interpretations. A second volume treats the generalized Grassmann product which constitutes an intermediate chain of products between the exterior and interior products, and applications to hypercomplex numbers and to mechanics. Other treatises with a more purely mathematical focus are [42] and [33].

\section{References}

1. G. BREDON, Topology and geometry, Graduate Texts in Mathematics, 139, Springer-Verlag, New York, 1993.

2. William E. BAYLIS, Clifford (Geometric) Algebras: With Applications in Physics, Mathematics, and Engineering, Springer, 1996.

3. Alexander Beilinson, Victor Ginzburg, and WOLFGANG SOERGEL, Koszul duality patterns in representation theory, J. Amer. Math. Soc., 9 (1996), no. 2 , 473-527.

4. Alexander A. Beilinson, Coherent sheaves on $\mathbb{p}^{n}$ and problems of linear algebra, Funct. Anal. Appl., 12 (1978), no. 3, 214-216.

5. JoHn Browne, Grassmann Algebra, vol. 1, Create Space Independent Publishing Platform, 2012.

6. WINFried BRUNS and JÜRgeN HERzog, CohenMacaulay Rings, Cambridge University Press, 1998.

7. Michael J. Crowe, A History of Vector Analysis: The Evolution of the Idea of a Vectorial System, Courier Dover Publications, 1967.

8. Christian Doran and ANTHONy LASEnBy, Geometric Algebra for Physicists, Cambridge University Press, 2007.

9. LeO DORST, Chris Doran, and JOAN LASEnby, Applications of Geometric Algebra in Computer Science and Engineering, Springer, 2002.

10. Leo Dorst, DANiEl FontiJne, and STEPHEN MANN, Geometric Algebra for Computer Science (Revised Edition): An Object-Oriented Approach to Geometry, Morgan Kaufmann, 2009.

11. LeO Dorst and JoAn LASEnby, Guide to Geometric Algebra in Practice, Springer, 2011.

12. D. EISENBUD et al., Computations in Algebraic Geometry with Macaulay 2, vol. 8, Springer, 2002.

13. D. Eisenbud, G. Fløystad, and F. O. Schreyer, Sheaf cohomology and free resolutions over exterior algebras, Trans. Amer. Math. Soc. 355 (2003), no. 11, 4397-4426.

14. DAVID EISENBUD, The Geometry of Syzygies: A Second Course in Algebraic Geometry and Commutative Algebra, vol. 229, Springer, 2005.

15. Michael FAlK, Combinatorial and algebraic structure in Orlik-Solomon algebras, European Journal of Combinatorics 22 (2001), no. 5, 687-698. 
16. G. FløYstad and J. E. VATne, (Bi-)Cohen-Macaulay simplicial complexes and their associated coherent sheaves, Comm. Algebra 33 (2005), no. 9, 3121-3136.

17. GUNNAR FLøYSTAD, Koszul duality and equivalences of categories, Trans. Amer. Math. Soc. 358 (2006), no. 6, 2373-2398.

18. W. FULTON and J. HARRIS, Representation Theory: A First Course, GTM 129, Springer, 1991.

19. D. J. H. GARLING, Clifford Algebras: An Introduction, vol. 78, Cambridge University Press, 2011.

20. HERMANN GRASSMANN and LLOYD C. KANNENBERG, Extension Theory, American Mathematical Society, 2000.

21. DANiEl R. GRAySON and Michael E. Stillman, Macaulay2, a software system for research in algebraic geometry, available http://www. math. uiuc.edu/Macaulay2/.

22. JÜRGEN HERZOG and TAKAYUKI HIBI, Monomial Ideals, Springer, 2011.

23. DAVID HeSTENES, Space-Time Algebra, Gordon and Breach, 1966.

24. Clifford Algebra to Geometric Algebra: A Unified Language for Mathematics and Physics, D. Reidel Publishing Company, 1984.

25. _ New Foundations for Classical Mechanics, Springer, 1999.

26. __ Old wine in new bottles: A new algebraic framework for computational geometry, Geometric Algebra with Applications in Science and Engineering, Springer, 2001, pp. 3-17.

27. DieTMAR Hildenbrand, Foundations of Geometric Algebra Computing, vol. 8, Springer, 2012.

28. B. J. Hiley and R. E. CALlaghan, Clifford algebras and the Dirac-Bohm quantum Hamilton-Jacobi equation, Foundations of Physics 42 (2012), no. 1, 192-208.

29. MeLvin HochSTER, Cohen-Macaulay rings, combinatorics, and simplicial complexes, Ring Theory, II (Proc. Second Conf., Univ. Oklahoma, Norman, Okla., 1975), Lecture Notes in Pure and Appl. Math., Vol. 26, Dekker, New York, 1977, pp. 171-223.

30. G. HORROCKS and D. MUMFORD, A rank two vector bundle on P4 with 15,000 symmetries, Toplogy, 12 (1973), no. $1,63-81$.

31. JAMES E. Humphreys, Introduction to Lie Algebras and Representation Theory, vol. 1980, Springer, New York, 1972.

32. Hongbo Li, David Hestenes, and Alyn Rockwood, Spherical conformal geometry with geometric algebra, Geometric Computing with Clifford Algebras, Springer, 2001, pp. 61-75.

33. DOUGLAS LUNDHOLM and LARS SVEnSSON, Clifford Algebra, Geometric Algebra, and Applications, 2009.

34. EZRA Miller and BERND STURMFels, Combinatorial Commutative Algebra, vol. 227, Springer, 2004.

35. JAmes R. Munkres, Elements of Algebraic Topology, vol. 2, Addison-Wesley, Reading, 1984.

36. Peter ORLIK and LOUIS SOlOMON, Combinatorics and topology of complements of hyperplanes, Invent. Math. 56 (1980), no. 2, 167-189.

37. Christian Perwass, Geometric Algebra with Applications in Engineering, vol. 4, Springer, 2008.

38. HANS-JOACHIM PETSCHE, AlBERT C. LEWIS, JÖRG LIESEN, and STEVE Russ (eds.), From Past to Future: Graßmann's Work in Context: Graßmann Bicentennial Conference, September 2009, Springer, 2010.
39. AleXANDer PolishchuK and LeONID Positselski, Quadratic Algebras, University Lecture Series, vol. 37, American Mathematical Society, 2005.

40. LeONid EFIMOVICH Positsel'SKII, Nonhomogeneous quadratic duality and curvature, Funct. Anal. Appl. 27 (1993), no. 3, 197-204.

41. GeRT SCHUBRING (ed.), Hermann Günther Graßmann (1809-1877): Visionary Mathematician, Scientist and Neohumanist Scholar, Kluwer Academic Publishers, 1997.

42. WILlIAM C. SCHULZ, Theory and application of Grassmann algebra, available at http://www. cefns.nau.edu/ schu1z/grassmann. pdf, 2011, preprint.

43. GERALD SOMMER, Geometric Computing with Clifford Algebras: Theoretical Foundations and Applications in Computer Vision and Robotics, Springer, 2001.

44. RiCHARD P. STANLEY, Combinatorics and Commutative Algebra, Birkhäuser Boston, 2004.

45. JoHn A. VINCE, Geometric Algebra for Computer Graphics, vol. 1, Springer, 2008.

46. Geometric Algebra: An Algebraic System for Computer Games and Animation, Springer, 2009.

47. S. A. YUZVINSKY, Orlik-Solomon algebras in algebra and topology, Russian Mathematical Surveys 56 (2007), no. 2, 293. 